\title{
Recovering from Work-related Strain and Stress with the Help of a Persuasive Mobile Application: Interview-based Thematic Analysis of Micro-entrepreneurs
}

\author{
Markku Kekkonen ${ }^{1}$, Harri Oinas-Kukkonen ${ }^{1}$, Eveliina Korkiakangas ${ }^{2}$ and Jaana Laiti- \\ nen $^{2}$ \\ ${ }^{1}$ University of Oulu, Faculty of Information Technology and Electrical Engineering, Oulu \\ Advanced Research on Service and Information Systems, 90570 Oulu, Finland \\ markku.kekkonen@oulu.fi and harri.oinas-kukkonen@oulu.fi \\ ${ }^{2}$ Finnish Institute of Occupational Health, P.O. Box 40, FI-00032 Työterveyslaitos \\ eveliina.korkiakangas@ttl.fi and jaana.laitinen@ttl.fi
}

\begin{abstract}
People often have long-term personal goals regarding health behavior change. Recently, the processes for achieving these goals have begun to be supported through behavior change support systems and especially through their persuasive software features. In a multidisciplinary research project focused on helping micro-entrepreneurs to recover from work-related strain and stress, a persuasive mobile application was developed. For gaining insights about the workings of the system and its persuasive features, we conducted 29 interviews with the system users. We used thematic analysis method with a deductive emphasis for analyzing the interviews. For some, concurrent usage of wearables or other applications led to discarding our application. Users thought that the application was relatively persuasive, but technical issues reduced its persuasiveness noticeably. When functioning properly, self-monitoring and reminders were found to be supportive for users to achieve their goals. Unobtrusiveness was found to increase the persuasiveness of reminders, while self-monitoring always seems to be dependent on the user's personal needs.
\end{abstract}

Keywords: Health, Behavior change, Recovery from work, Goal setting, Mobile application, Persuasive Systems Design, Self-monitoring, Reminders, Thematic analysis.

\section{Introduction}

Modern health information technologies can provide cost-effective solutions for improved healthcare [1]. Such technologies can be successfully adopted into use by healthcare providers and professionals even for seeking to change old routines into new and more efficient ones [2]. The digital transformation of healthcare is not a silver bullet void of challenges, however [1]. For example, health behavior interventions utilizing information technology can be less effective if they do not incorporate evidence-based approaches and behavior change theories effectively [3]. Technologically delivered health solutions, especially mobile-based, can also potentially change 
healthcare for those, who currently have poor access to it [4]. Mobile health solutions can influence health behaviors of large population segments [3].

In Promo@Work research project, a Health Behavior Change Support System (HBCSS) [5], known as 'Recover!' was developed. The aim of the system was to help entrepreneurs to recover from work-related strain and stress. The system was implemented as a native mobile application for Android operating system. The target users, micro-entrepreneurs, are a special group of entrepreneurs who employ less than 10 persons while having an annual turnover less than 2 million EUR [6]. Microentrepreneurs, a large segment of population, may often lack proper occupational healthcare.

The application was developed in collaboration with a multidisciplinary team including entrepreneurship researchers, social psychologists and occupational healthcare professionals. Persuasive Systems Design (PSD) model [7], Selfdetermination theory (SDT) [8] and an adaptation of the Transtheoretical model (TTM) [9] were utilized in the design and development process.

A randomized controlled trial was successfully conducted. After two-month intervention period, we sought out to interview the intervention group participants about the usage of the application, their experiences and achieving of goals.

The research question for this paper is as follows: In the case of goal setting, how and to what extent did the users' goals change during the usage period and whether the PSD features supported the users in achieving their goals?

The outline for the paper is as follows. The system and research method will be described in the 'Study setting' section. The findings will be presented and interpreted in 'Results'. In 'Discussion', the practical and theoretical implications will be discussed, in addition to limitations. The final section will be 'Conclusions'.

\section{$2 \quad$ Study setting}

\subsection{The HBCSS}

The developed HBCSS was theory-based. SDT was chosen as the theoretical model based on the reported effectiveness regarding behavior change interventions [10]. TTM was adopted for goal setting because of the pragmatism of the 'stages-ofchange' approach. Additionally, it was suited for research in this case, as there was no need for complex approaches, thus a simplified adaption of TTM was sufficient. In addition to personal goals the users might have had, the application offered support for individual goal setting via tasks set for the user. Before undertaking any tasks within the app, users had to choose one of the three goals adapted from TTM: Thinking and observing, Acting and doing, or Maintaining. The tasks in each goal could be undertaken on the spot e.g. reading a short text about a health topic while answering self-reflective questions; or were ones that required activities during a longer period while requiring the usage of self-monitoring tools of the HBCSS.

The persuasive features of the system were carefully analyzed and decided together by the research consortium by using the PSD model. The persuasive features included goal setting and the principles of self-monitoring, rehearsal, praise, reminders, sug- 
gestion, liking, trustworthiness and social comparison [7]. In the PSD model, both self-monitoring (primary task support) and reminders (dialogue support) are specifically tied to achieving goals. In addition to these, all features in the primary task support category are to varying extents linked to goal setting and achieving set goals.

The HBCSS under investigation contained seven health problem domain modules. Each module contained one or more self-monitoring tools (see table 1). The main role of the tools was to allow the users to measure and monitor their self-perceived situation in relation to health behaviors. Additionally, the tools were also meant to be selfreflective, thus allowing the users to reflect on the behaviors that may lead to health choices [11]. Some of the self-monitoring tools might be considered as having additional functions, in addition to self-monitoring. For example, the posture change reminder not only reminded the users to change posture, but also allowed the users to reflect whether they had not moved for 30 or 60 minutes.

Table 1. Health problem domains and self-monitoring tools in the application.

\begin{tabular}{ll}
\hline Self-monitoring software feature & Health problem domain \\
\hline Stress statistics & Stress management \\
Pedometer & Exercising \\
Recovery statistics & Recovery from work \\
Posture change reminder & Sedentary behavior \\
Dietary rhythm rehearsal & Nutrition (dietary behavior) \\
Diet planning & Nutrition (dietary behavior) \\
Circadian rhythm & Sleep, Efficient working hours (time \\
\hline
\end{tabular}

Both tools at the nutrition category (dietary rhythm rehearsal and diet planning) were inherently about virtual rehearsal, but as one could check the plans for both diet and rhythm, they served also as self-monitoring tools. Regarding the statistic tools, both were self-reflective in nature, as you could monitor your daily or weekly level of selfperceived stress/recovery. Additionally, both also incorporated aspects of social comparison, as users could also compare their own level to the average level of the user base. Circadian rhythm tool allowed users to monitor the proportion of work, free time, and sleep within 24-hour timeframe.

The second goal setting related persuasive software feature studied here, reminders, had two main roles in the application: (1) Once a week the users received a push notification reminding them to fill a quick weekly survey, thus also reminding them to use the application. (2) Whenever users chose tasks requiring longer periods for completion, a push notification was send after a day (or few days, depending on the goal and task), thus reminding the users that they had a task pending.

\subsection{Research method}

We decided to take a closer look at the goals of the participants, and the premises, validity and role of the two aforementioned principles in achieving goals. Therefore, 
we conducted a deductive thematic analysis from the transcribed interviews. Among qualitative research, interviews are the most common method for collecting data [12].

According to Braun and Clarke [13], "Thematic analysis is a method for identifying, analyzing and reporting patterns (themes) within data. It minimally organizes and describes your data set in (rich) detail". We chose a deductive approach for this thematic analysis, because we aimed to test the PSD principles of self-monitoring and reminders in this particular case [14]. Deductive thematic analysis, however, tends to produce less rich overall description of the data set, but is suited for answering theorybound research questions, as it is analyst-driven, i.e. driven by analytical or theoretical interest; and the analysis can be more detailed for some aspects of the data [13].

Data collection. The interviews were conducted as semi-structured, with a predefined set of open-ended questions. When deemed necessary, the interviewer could ask follow-up questions for in-depth answers. Question sets had been used in a previous intervention study [15], in which the team had participated, and the questions were piloted with two pilot participants for this intervention study.

When piloting the application, it was discovered that recruiting interviewees via email could be difficult, as most pilot users never answered our emails. Therefore, we decided to ask for a voluntary pre-agreement for interview in the enrollment phase of the intervention, which required those interested to fill in their phone numbers. Some other researchers in the research consortium were also interviewing other participants from a different point of view. All those who had expressed consent to be interviewed, were randomized and split into two lists, one for each group of researchers. The list for our team consisted of 50 randomized participant names and contact info.

Each participant on the list was tried to be reached by a phone call three times, while dividing the calls into several days and potentially suitable times. In case a participant did not answer these calls, we send an email or mobile text message explaining why we had called, which led to some calling back to us. Total of 32 participants were recruited for our study to be interviewed about their experiences regarding the application. Three interviewees canceled for personal reasons; therefore, we conducted 29 interviews. As we also collected log data about the actual usage of the application, we checked that all invited interviewees had logged into the application.

The main platform used for the interviews was Skype, a Voice over Internet Protocol (VoIP) software. Only the audio connection was enabled and thus recorded. The interviewees were asked for their final consent in the beginning of the interviews and each had received the information about the research beforehand. In case the interviewees shunned using Skype, recorded telephone calls were used instead.

The recorded interviews were transcribed into textual form for analysis purposes. The content of the interviews were not altered in any way for the transcriptions, e.g. dialects were transcribed precisely, but the transcriptions were not precise phonetically, meaning that for example not all coughs or sneezes were marked down explicitly.

Data analysis. In the first phase of thematic analysis, researchers should familiarize themselves with the data, while in the second phase, initial coding of the data takes place [13, 16], potentially with the help of computer program software [17] suited for the process. The third phase consists of searching for themes within the coded data and once themes are devised, the themes are reviewed in the fourth phase 
$[13,16]$. Definition and naming takes place in the fifth phase $[13,16]$, while making sure that all text relevant for the research question are included in the themes, but avoiding the pitfall of refining the themes forever [17]. In the sixth phase, a final thematic analysis is written $[13,16]$, while using direct quotes from the participants as examples in the report [17]. Making notes and/or marking ideas for coding in the first phase of analysis can be beneficial later on [13]. Keeping a reflexivity journal during the process increases the trustworthiness of the research [16].

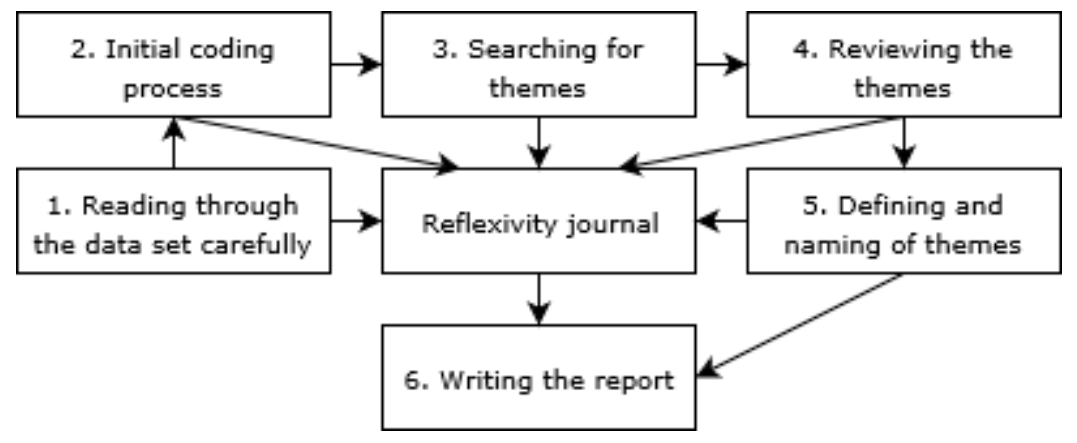

Fig. 1. The data analysis process

At first, the transcribed interviews were read through carefully, while paying attention to information, such as names and places of living, which could be used to recognize the interviewees, thus needing to be shrouded in order to grant the interviewees anonymousness. A reflexivity journal was initiated concurrently. Next, the interviews were carefully re-read twice. After the data set familiarization phase, the interviews were printed on paper and initial codes were generated. At this point, the coding was done manually by using colored highlighter pens and by writing notes [13].

After the initial coding, we decided to use computer software program NVivo version 11 (by QSR International) for the next phase. Therefore, the transcribed interview files were exported into NVivo and the prints were used for guidance for transferring the initial codes into the program. Because we had chosen a deductive approach, we formed the main themes (parent nodes in NVivo) based on the research question. Memos linked to each main theme were created in NVivo at this point. Subthemes were formed in similar way and presented as child nodes in NVivo.

The themes were reviewed, which involved deleting unnecessary codes as well as those overlapping with other codes. In the next phase, the themes were defined and named. In the final phase, a report of the findings and a final analysis was written, which will be presented in the 'results' section. The reflexivity journal in the form of memos and notes was helpful for presenting and interpreting the results (see figure 1).

\section{Results}

Reactions to the application were mostly positive, although many complained about technical difficulties hindering their usage. Total of 21 interviewees reported that the 
application was persuasive (believable and convincing) for them, whereas eight interviewees thought that it was not that persuasive in their case. Nevertheless, the application was deemed persuasive by over two thirds of the interviewees, including many of those complaining about the technical difficulties, as they expressed that it would be even more persuasive if it would function correctly. As regarding persuasiveness, the aspect was brought up in two different questions in the interviews, and few interviewees asked for a clarification, which was given for them, Most of the interviewees expressed interest in an updated and bug-free version of the application, giving further evidence that the application was found useful and engaging, thus also persuasive.

During the interviews, the users were asked about their personal goals and had their personal goal changed or remained the same after the usage period. The themes for goal setting were formed according to the research question (see figure 2).

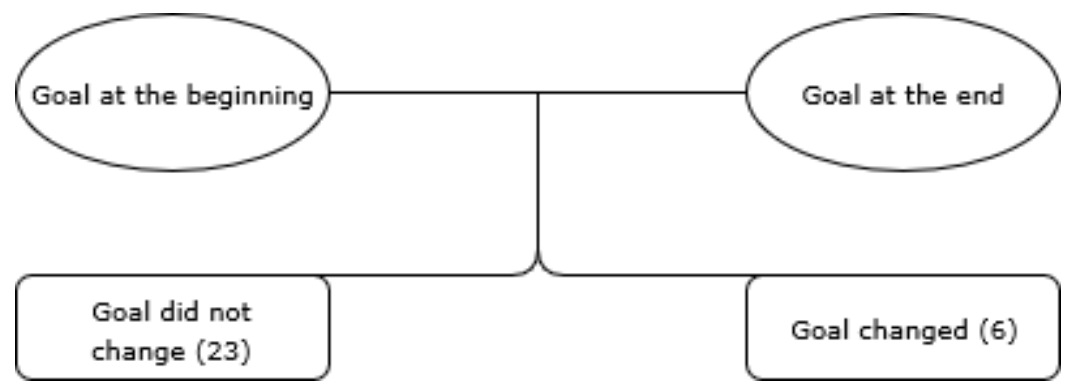

Fig. 2. Themes for goal setting

We planned to ask the interviewees about reminders, but the subject actually came up in most of the interviews, regardless whether we would have asked the prepared question or not. Only with the case of one user, we did not ask about reminders due to low usage of the app and neither did the subject come up by the interviewee.

We did not ask any specific questions about self-monitoring, but the subject came up frequently. The themes for persuasive features investigated here (self-monitoring and reminders, see figure 3) were formed according to the research question.

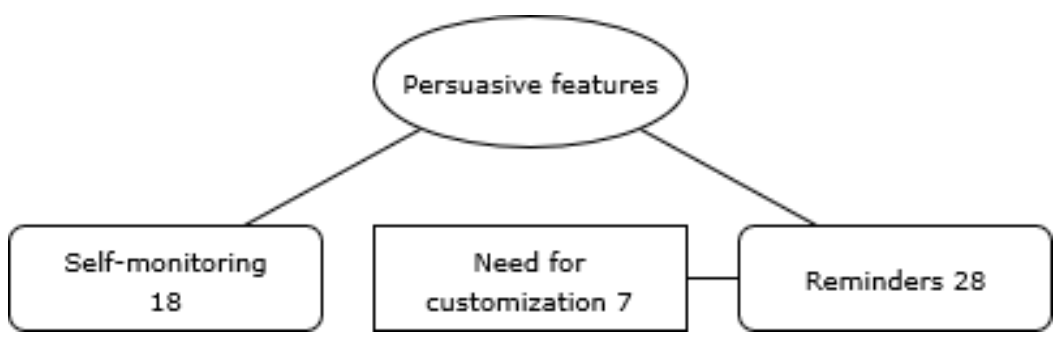

Fig. 3. Themes for persuasive features tested

The only exception was one subtheme (customization), which was formed inductively. Combining deductive and inductive approaches is not an uncommon phenomenon in thematic analysis [16, 18-19]. 


\subsection{Personal goal}

Goal at the beginning of usage. A recurring pattern could be spotted from the interviews: all participants either had problems with their well-being or concerns about their health. Both issues were intertwining with the core of the intervention, which was recovery from work-related strain and stress. The motivation for enrollment might have differed, as for some it was curiosity:

"Well, mostly I came along because of interest, as I should do something about my own well-being and occupational well-being, so it hit the spot. When thinking about having enough strength to keep on working, mostly the goal was that working life and spare time would be in some sort of balance, so maybe having more free time and gaining tools for balancing it" (Interview 16).

For others, there might have been more severe need for help, but everyone had obviously realized that they had to do something about their situation eventually:

"I had an idea about somehow getting something new, which would help me to get by, because at the moment I'm working on the brink of exhaustion" (Interview 13).

Personal goal at the end of usage. It should be noted that several interviewees used the application for shorter than two months due to various reasons, which led them to not use the application regularly. Five users stopped using the application altogether almost immediately after the beginning of the intervention. The early abandonment of the application was mostly due to technical issues experienced or from being too busy due to work. Nonetheless, 15 users reported that the application had clearly supported or affected their personal goal in one way or another:

"My goals are the same as before, so the app has not changed them, but it has been a part of supporting the orientation to achieving the goals with time" (Interview 17).

Goal did not change. For 23 interviewees, the goal remained practically the same at the end of usage. Nevertheless, even if remaining the same, the goal or idea of the goal had been strengthened for some users:

"The application, it also fortifies the confirmation that I just have to take the time for exercising. All those fancy goals regarding exercising in the app those might not never truly be reality for me as such, but if adapted, then they might" (Interview 15).

Achieving personal goals might be hard and take a lot of time, especially if one tries to change a behavior that has been going on for years. Even with the support and coaching of a persuasive application, it could be difficult, if one does not want to take any time off from work. Being addicted to work bears down to such fundamental issues that no application alone could resolve:

"The goals are more or less the same, because it is hard [to achieve them], when one is a so-called workaholic, which has been going on for many years as an entrepreneur. Therefore, changing habits is not easy" (Interview 9). 
Goal changed. For six interviewees, the goal appeared to have changed beyond the point of fortification up to expansion or refinement. Achieving personal health-related goals might take years, so we held no misconception about the application miraculously curing all sort of ailments during a two-month usage period. Nevertheless, selfreflection [11] was one of the key issues in the application, therefore it comes as no surprise that people who take the time to reflect their own behavior might have their goals at least refined or expanded, if not achieved:

"I now have a [new] goal as I have to get my sleep [quality and quantity] in order and here [in the application] is help for that" (Interview 3).

For many interviewees, the reason for enrolling into the intervention was the need to do something about their health, so they might have been pondering their personal health issues for years. In that sense, even starting to use or trying out the application might have been an important factor in the long road to better health behavior:

"Therefore, if my goal has changed, it has only been to the better direction, because when I was busy and stressed, which I had been for many years, I thought that eventually I would die. I thought that now I just have to start taking better care of myself and that has only increased. Whether the application had a part in that, it certainly did not decrease my need, so yes, it was a small part in the process" (Interview 19).

\subsection{Self-monitoring}

As described in the PSD model [7], "A system that keeps track of one's own performance or status supports the user in achieving goals". The application was a system that enabled self-monitoring; therefore, the application should have supported the users in achieving goals. In this case, it could be said that the self-monitoring tools were there to help the users in the three-level TTM-based task related goals, rather than directly with their personal goals. Nevertheless, this did not exclude the tools being used by the users for supporting their personal goals:

"Well now, that pedometer is by definition a good thing, because I became aware of the fact that as I am doing [sedentary] work at the office, I get really too few steps during the day. At least to that it has helped, so maybe because of it I have paid more attention now when following the amount of steps" (Interview 16).

Some of the interviewees reported using wearables (for self-monitoring) concurrently with the application, or even abandoning the application in favor of wearables or other health applications with sensor-based real-time self-monitoring functions. Previous experiences with health applications seemed to have molded the expectations for them, which might have led to disappointments, as the app did not have various sensor-based measuring functions. Few had started using wearables or another application more or less after the intervention period begun. Some thought that it would have been nice to be able to connect their wearables into the 'Recover!'application, but as they could not, they continued to use both: 
"Now I have actually been testing this bracelet or watch, which follows my heartbeat and sleep and so on automatically. I don't have to mark those up myself, so I have used it for about a month now, but it doesn't mean that I wouldn't use the application, because there was that other stuff in there" (Interview 26).

It seemed that the interviewees chose whether to use the self-monitoring tools of the app, or those provided by other applications or wearable devices, based on their personal needs or situations. Lack of comprehensive set of sensor-based selfmonitoring tools did not bother everyone, as they were more or less satisfied with the tools provided by the application. This was likely because the tools, such as the circadian rhythm, suited their needs. Nevertheless, the app had an automatic, sensor-based pedometer, which was used by many interviewees. All the other tools were interactive and required actions from the user. Rather than being merely self-monitoring, the tools were also intended to be self-reflective:

"In a way, I had this conception of how these things are, but when you really start marking them and following them, it might be that the truth really is not what you thought it to be" (Interview 26).

One interviewee complained that the application and the circadian rhythm tool were too persuasive in the sense that the user felt like being a slave to the phone, constantly interacting with it. This led to the user abandoning the app due to stress, so apparently the self-monitoring tool did not suit the user's current health problem that well, as the user also complained about being busy and stressed otherwise. However, the aforementioned user also thought that the application was still partially responsible for changing the personal goal in a positive way. Few others complained about minor issues with the self-monitoring tools, mostly with pedometer, of which one issue was a major bug (app crashing when turning phone sideways):

"There were good systems, pedometer and such, but if I went for a jog and the phone turned sideways [while jogging], it rather became an obstacle" (Interview 11).

It could be said that in the case of the application, the premises were true if the user had a need for the types of tools provided by the application and the tools functioned technically. Therefore, if the premises were true, then the argument was also solid, thus the application could be said supporting the users, whether they achieved their goals during the usage or not.

\subsection{Reminders}

As described in the PSD model [7], "If a system reminds users of their target behavior, the users will more likely achieve their goals". The application was a system that utilized reminders, so therefore the users of the application should have been more likely to achieve their goals. It should be noted that many users had some technical difficulties with the reminders, although it should also be mentioned that some of the difficulties reported might rather have been misconceptions by the users (than actual bugs). For example, one user thought that the reminders regarding tasks were mal- 
functioning for not getting any. The user for some reason had assumed that tasks would be given for the users automatically, which was not the case. Nevertheless, when functioning properly, the reminders were seen as helpful for achieving the goals set:

"[Reminders] were good, because otherwise those [tasks] would be left undone, so it is very good that there comes reminders [from the application], because if there are not reminders, then you just will not remember" (Interview 15).

However, there were other opinions, as not all users were keen on having reminders, at least not very often. If not using the application actively, the reminders might have been felt as annoying. Even if the user knew and admitted that reminders were useful and supportive, they might still have been felt as annoying. One solution to dissolving potential annoyance of reminders could very well be customization:

"When you know that I've set this reminder myself, just like you put alarms in a calendar, I must say that those aren't annoying at all when they are something that I've set there myself, so that would be a good idea" (Interview 17).

Some users felt that the reminders occasionally arrived at improper times, such as when they had meetings with customers. Therefore, it was difficult for them to react to reminders at due times. According to the sixth postulate of the PSD model [7], persuasive features at improper moments may lead to undesirable outcomes and in the case of reminders, ending up ignored. Customization could also be a solution for avoiding the possible obtrusiveness of reminders:

"I cannot have any reminders beeping in those situations [with customers], so for example it would be proper only on those days that I am at the office, which are limited. I noticed, that I could not set a [customized] reminder in this app" (Interview 6).

It could be said that in the case of the application, the premises were true if: (1) the reminders were not annoying, or obtrusive, and (2) the reminders functioned technically. Therefore, if the premises were true, then the argument was also solid, thus the users could have been said to be more likely to achieve their goals, whether they achieved them during the usage or not.

\section{Discussion}

The long-term personal goals of participants might have been under the way for a while. Fifteen users reported that the application had clearly supported or affected their personal goal, of which six users thought that their goal had changed during the relatively short usage period. Hence, it could be seen as quite a positive thing that over a half of the users felt that their personal goals were fortified and perhaps even refined or expanded during the usage process.

Commercial systems supporting behavior change might nowadays be depending heavily on sensor based self-monitoring tools, as well as on external wearable devices. This seemed to have molded the expectations of some of the interviewed partici- 
pants, which evidently affected the usage. Pedometer was the only sensor based selfmonitoring tool in the application, which for some was enough, but for others insufficient as they were clearly expecting more. Nevertheless, those using the selfmonitoring tools of the application, whether the pedometer or the non-sensor based options e.g. circadian rhythm tool, seemed to benefit from the self-reflective side of the tools. On the other hand, if the users could not find tools suitable for their specific needs, they could still use the application by going through the contents and completing the tasks. They seemed to switch to using the tools of other applications or wearable devices for self-monitoring purposes in the aforementioned case, which in turn seemed to decrease the overall interest for the 'Recover!'-application for those users.

As regarding reminders, customization could be a solution for avoiding annoying the users, as well as avoiding obtrusiveness. Even if push notifications at improper times would not be bothersome for all, they seemed to disturb some. Implementing full customization (times and dates) for reminders (push notifications) could consume resources. At minimum, it might be wise to allow the option of turning the reminders off altogether.

The contents of the application were planned specifically for micro-entrepreneurs, but as they were derived from general evidence-based health and well-being recommendations, they were also largely applicable for other entrepreneurs as well as for the general population.

As regarding the limitations, this study was about one application in one context. Additional limitations of this study include that the results were based on the interviews, which were more or less subjective opinions of the users, although important as portrayals of personal experiences. Analyzing application's actual usage by using log data could add value to analyses such as the one carried out here.

\section{Conclusions}

Interviews can be fruitful for gaining insights about users' goals, system's persuasiveness, and its persuasive features. Users may have long-term goals, which persuasive technologies can offer support. Nevertheless, it is a challenge for design that some users may perceive a persuasive feature as fine, whereas others may find it irritating or useless. This is especially challenging when designing for very heterogeneous target groups. In any case, software bugs tend to decrease the persuasiveness and overall trustworthiness dramatically.

For designers, this study offers user-based insights and perceptions about selfmonitoring tools and customization of reminders. For researchers, this study offers further evidence that self-monitoring and reminders can indeed support users, as long as the premises are true.

Acknowledgements. We wish to thank our colleagues at Promo@Work research project, funded by the Strategic Research Council at the Academy of Finland, contract no. 303430 (Finnish Institute of Occupational Health) and contract no. 303431 (University of Oulu, OASIS). 


\section{References}

1. Agarwal, R., Gao, G., DesRoches, C., Jha, A.: Research commentary-The digital transformation of healthcare: Current status and the road ahead. Information Systems Research 21(4):796-809 (2010)

2. Goh, J.M., Gao, G., Agarwal, R.: Evolving work routines: Adaptive routinization of information technology in healthcare. Information Systems Research 22(3):565-585 (2011)

3. Borrelli, B., Ritterband, L.M.: Special issue on eHealth and mHealth: Challenges and future directions for assessment, treatment, and dissemination. Health Psychology 34(S):1205 (2015)

4. Meier, C.A., Fitzgerald, M.C., Smith, J.M.: eHealth: extending, enhancing, and evolving health care. Annu Rev Biomed Eng 15:359-382 (2013)

5. Oinas-Kukkonen, H.: A foundation for the study of behavior change support systems. Personal and Ubiquitous Computing, 17(6), 1223-1235 (2013)

6. European Commission: Commission Recommendation of 6 May 2003 concerning the definition of micro, small and medium-sized enterprises. Official Journal of the European Union, 46, (2003).

7. Oinas-Kukkonen, H., Harjumaa, M.: Persuasive systems design: Key issues, process model, and system features. Communications of the Association for Information Systems 24(1):28 (2009)

8. Ryan, R.M., Deci, E.L.: Self-determination theory and the facilitation of intrinsic motivation, social development, and well-being. Am Psychol 55(1):68 (2000)

9. Prochaska, J.O., DiClemente, C.C.: The transtheoretical approach. Handbook of psychotherapy integration 2:147-171 (2005)

10. Ng, J.Y., Ntoumanis, N., Thøgersen-Ntoumani, C., Deci, E.L., Ryan, R.M, Duda, J.L., Williams, G.C.: Self-Determination Theory Applied to Health Contexts: A Meta-Analysis. Perspectives on Psychological Science 7. 325-340 (2012)

11. Halttu, K., Oinas-Kukkonen, H.: Persuading to reflect: role of reflection and insight in persuasive systems design for physical health. Human-Computer Interaction 32(5-6), (2017)

12. King, N.: Using interviews in qualitative research. In: Cassel, C., Gillian, S.: Essential Guide to Qualitative Methods in Organizational Research. SAGE. London (2004)

13. Braun, V., Clarke, V.: Using thematic analysis in psychology. Qualitative research in psychology 3(2):77-101 (2006)

14. Vaismoradi, M., Turunen, H., Bondas, T.: Content analysis and thematic analysis: Implications for conducting a qualitative descriptive study. Nurs Health Sci 15(3):398-405 (2013)

15. Karppinen, P., Oinas-Kukkonen, H., Alahäivälä, T., Jokelainen, T., Keränen, A.-M., Salonurmi, T., Savolainen, M.: Persuasive User Experience in Health Behavior Change Support System: A 12-month Study for Prevention of Metabolic Syndrome. International Journal of Medical Informatics, 96, (2016), pp. 51-61.

16. Nowell, L.S., Norris, J.M., White, D.E., Moules, N.J.: Thematic analysis: Striving to meet the trustworthiness criteria. International Journal of Qualitative Methods 16(1):1609406917733847 (2017)

17. King, N.: Using templates in the thematic analysis of text. In: Cassel, C., Gillian, S.: Essential Guide to Qualitative Methods in Organizational Research. SAGE. London (2004)

18. Fereday, J., Muir-Cochrane, E.: Demonstrating rigor using thematic analysis: A hybrid approach of inductive and deductive coding and theme development. International journal of qualitative methods 5(1):80-92 (2006)

19. Gregory, R., Muntermann, J.: Theorizing in design science research: inductive versus deductive approaches. ICIS 2011 Proceedings (2011) 